\title{
Genotoxic, neurotoxic and neuroprotective activities of apomorphine and its oxidized derivative 8-oxo-apomorphine
}

\footnotetext{
J.N. Picada ${ }^{1,2}$,

R. Roesler ${ }^{2,3}$ and J.A.P. Henriques ${ }^{1,2}$
}

\author{
${ }^{1}$ Curso de Farmácia, Universidade Luterana do Brasil, Canoas, RS, Brasil \\ ${ }^{2}$ Centro de Biotecnologia e Departamento de Biofísica, and \\ ${ }^{3}$ Departamento de Farmacologia, Instituto de Ciências Básicas da Saúde, \\ Universidade Federal do Rio Grande do Sul, Porto Alegre, RS, Brasil
}

\section{Correspondence \\ J.A.P. Henriques \\ Centro de Biotecnologia, UFRGS \\ Av. Ipiranga, 9500, Campus do Vale \\ 91501-970 Porto Alegre, RS \\ Brasil \\ Fax: +55-51-3316-6084 \\ E-mail: pegas@dna.cbiot.ufrgs.br}

Presented at the XI Congresso Brasileiro de Biologia Celular, Campinas, SP, Brazil, July 15-18, 2004.

Research supported by FAPERGS, CAPES, CNPq, and GENOTOX

(Genotoxicity Laboratory, UFRGS).

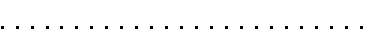

Received July 16, 2004

Accepted January 6, 2005

\begin{abstract}
Apomorphine is a dopamine receptor agonist proposed to be a neuroprotective agent in the treatment of patients with Parkinson's disease. Both in vivo and in vitro studies have shown that apomorphine displays both antioxidant and pro-oxidant actions, and might have either neuroprotective or neurotoxic effects on the central nervous system. Some of the neurotoxic effects of apomorphine are mediated by its oxidation derivatives. In the present review, we discuss recent studies from our laboratory in which the molecular, cellular and neurobehavioral effects of apomorphine and its oxidized derivative, 8-oxo-apomorphine-semiquinone (8-OASQ), were evaluated in different experimental models, i.e., in vitro genotoxicity in Salmonellal microsome assay and WP2 Mutoxitest, sensitivity assay in Saccharomyces cerevisiae, neurobehavioral procedures (inhibition avoidance task, open field behavior, and habituation) in rats, stereotyped behavior in mice, and Comet assay and oxidative stress analyses in mouse brain. Our results show that apomorphine and 8-OASQ induce differential mutagenic, neurochemical and neurobehavioral effects. 8-OASQ displays cytotoxic effects and oxidative and frameshift mutagenic activities, while apomorphine shows antimutagenic and antioxidant effects in vitro. 8-OASQ induces a significant increase of DNA damage in mouse brain tissue. Both apomorphine and 8-OASQ impair memory for aversive training in rats, although the two drugs showed a different dose-response pattern. 8-OASQ fails to induce stereotyped behaviors in mice. The implications of these findings are discussed in the light of evidence from studies by other groups. We propose that the neuroprotective and neurotoxic effects of dopamine agonists might be mediated, in part, by their oxidized metabolites.
\end{abstract}

Key words

- Apomorphine

- 8-Oxo-apomorphinesemiquinone

- Dopamine

- Genotoxicity

- Neurotoxicity 


\section{Introduction}

The primary pathology characterizing Parkinson's disease (PD) is a selective degeneration of dopaminergic neurons in the substantia nigra pars compacta, which project mainly to the striatum (1). It has been proposed that oxidative stress plays a pivotal role in the neurodegenerative damage associated with PD (1-6). For instance, reduced glutathione levels (7) increased lipid peroxidation in the substantia nigra (8), and oxidative DNA damage $(9,10)$ have been suggested to be involved in the neurodegenerative mechanisms underlying PD.

Apomorphine [4H-dibenzo $(d e, \mathrm{~g})$ quinoline-10,11-diol, 5,6,6a,7-tetrahydro-6-meth$\mathrm{yl}-(\mathrm{R})]$ is a potent D1 and D2 dopamine receptor agonist that rapidly enters the brain and accumulates in the striatum (11). Apomorphine displays antiparkinsonian properties similar to those of L-DOPA and has been shown to be useful for treating PD patients, especially in the late stages of the disease (12-14). Increasing evidence suggests that some of the effects of dopamine and dopamine receptor agonists might be mediated by their quinone and semiquinone oxidation derivatives (15-19). Dopamine's oxidation derivatives such as dopamine o-quinone and o-semiquinone occur in the normal brain (20). Dopamine, apomorphine, and L-DOPA easily autoxidize, producing quinone and semiquinone derivatives that may lead to the formation of toxic products and superoxide radicals (15-19). The oxidation-related properties of apomorphine lead to apparently paradoxical activities of the drug, which may act either as an antioxidant or as a prooxidant (reviewed in Ref. 21). Ubeda et al. (22) reported that apomorphine can act as a pro-oxidant, leading to DNA damage and to deoxyribose degradation induced by $\mathrm{Fe}^{3+}$ and $\mathrm{Cu}^{2+}$ by a mechanism related to the generation of superoxide radicals. In contrast, apomorphine has been shown to inhibit membrane lipid peroxidation in vitro and in vivo (23). In addition, toxic effects of apomorphine to cultured neurons have been shown to correlate with its autoxidation (19).

Since increasing evidence suggests that some of the neuronal effects of dopamine, LDOPA, and apomorphine are mediated by their oxidation derivatives $(16,19)$, we propose that the investigation of the neurobiological actions of isolated oxidation derivatives of apomorphine is clinically relevant and might help to elucidate the mechanisms involved in oxidative stress-mediated neuronal damage in schizophrenia (18), neurodegeneration associated with $\mathrm{PD}$, and the effects of dopaminergic therapies in PD patients. We described the isolation of an apomorphine autoxidation semiquinone derivative, 8-oxo-apomorphine-semiquinone (8OASQ), and demonstrated for the first time its mutagenic activity in vitro $(24,25)$.

\section{Mutagenicity}

Little was known until recently about the genotoxic effect of apomorphine. It has been shown to induce frameshift mutations in Salmonella typhimurium TA1537 and this mutagenic action was attributed to its oxidation to mutagenic products (26). The clastogenic activity of apomorphine has been demonstrated in a Chinese hamster lung cell line in the absence of metabolic activation (S9 mix) (27).

In vitro genotoxicity assays have shown that apomorphine and its oxidation derivative 8-OASQ induce frameshift mutations in TA98 and TA97 S. typhimurium strains, with 8-OASQ being up to two times more mutagenic (Table 1) (25). The ability of the oxidized apomorphine form, 8-OASQ, to induce larger quantities of frameshift mutations than apomorphine may reside in its more aromatic and planar structure, a feature of quinone compounds $(28,29)$. This presumably favors intercalation into DNA, hence promoting frameshift mutations. However, for strains which detect oxidative mutagens, 
TA102 S. typhimurium strain, and IC188 and IC203 E. coli strains, 8-OASQ acted as a mutagen. This action was probably mediated by pro-oxidant effects that induced DNA oxidative damage, while apomorphine was an antimutagen, inhibiting by up to $80 \%$ hydrogen peroxide $\left(\mathrm{H}_{2} \mathrm{O}_{2}\right)$ and t-butylhydroperoxide (t-BOOH)-induced mutagenicity in all strains, possibly due to an antioxidant property (25). Compounds with a catechol structure such as apomorphine have metal chelating properties and can act as reducing agents and radical scavengers (30). However, as a reducing agent, apomorphine can also contribute to the generation of highly toxic hydroxyl radical by maintaining iron in the ferrous state. The overall mechanism by which antioxidant drugs affect the level of reactive oxygen species (ROS) depends on the balance between radical scavenging and radical activating properties (23).

In sensitivity assays with 10 strains of the yeast Saccharomyces cerevisiae apomorphine was only clearly cytotoxic to some strains at higher doses, whereas 8-OASQ dose-dependently sensitized all strains, especially the mutants lacking catalase $(\Delta c t t 1)$, superoxide dismutase $(\Delta \operatorname{sod} 1)$ and Yap1 transcription factor ( $\triangle$ yap 1$)$, suggesting that 8-OASQ cytotoxicity towards $S$. cerevisiae results from its pro-oxidant properties (25). Furthermore, apomorphine tended to protect $S$. cerevisiae strains against oxidative damage induced by high concentrations of $\mathrm{H}_{2} \mathrm{O}_{2}$ and $\mathrm{t}-\mathrm{BOOH}$, while 8-OASQ enhanced the pro-oxidant effects and induced adaptation responses to these agents.

\section{Neurotoxicity}

Previous studies have shown that the autoxidation products of apomorphine and other catechols (e.g., dopamine) might lead to deleterious effects on neuronal cells and neural function (16). The cytotoxic effects of apomorphine on cultured neurons have also been shown to correlate with its autoxidation prod- ucts (31). El-Bachá et al. (19) showed that apomorphine promotes necrosis in rat glioma C6 cells through the formation of ROS, quinones and a melanin-like pigment during autoxidation. Both apomorphine autoxidation and cell damage were prevented by thiols.

\section{Neurobehavioral toxicity}

The possible neurotoxic effects of apomorphine and 8-OASQ have also been evaluated using behavioral endpoints of neural function (32). Both apomorphine and 8OASQ impaired memory for aversive training in rats, although the two drugs showed a different dose-response pattern. Apomorphine, but not 8-OASQ, dose-dependently impaired habituation, a type of nonaversive, nonassociative memory. Neither apomorphine nor 8-OASQ affected nociception, locomotion or exploratory behavior. The differential behavioral deficits induced by apo-

Table 1. Mutagenic effects of apomorphine and 8-oxo-apomorphine-semiquinone (8OASQ) in bacterial strains detecting frameshift ( $S$. typhimurium TA98 and TA97 strains) and oxidative mutagens (S. typhimurium TA102 strain and E. coli IC188 and IC203 strains), without metabolic activation (S9 mix)

\begin{tabular}{lccccc}
\hline Drugs and doses $(\mu \mathrm{g} /$ plate) & \multicolumn{5}{c}{ Mutagenic index } \\
\cline { 2 - 5 } & TA98 & TA97 & TA102 & IC188 & IC203 \\
\cline { 2 - 5 } & & & & \\
Apomorphine & NT & NT & 1.1 & 1.0 & 1.0 \\
10 & 1.3 & 1.8 & 1.2 & 0.8 & 0.8 \\
20 & 1.5 & 2.5 & 1.2 & 0.7 & 0.6 \\
40 & 2.1 & 3.0 & 1.5 & 0.5 & 0.7 \\
60 & 2.4 & 4.1 & NT & NT & NT \\
80 & + & + & - & - & - \\
Result & & & & 1.3 & 1.6 \\
8 -OASO & 2.1 & 3.1 & 1.1 & 1.3 & 2.2 \\
10 & 2.8 & 4.8 & 1.0 & 1.9 & 4.1 \\
20 & 3.5 & 6.2 & 1.0 & 2.6 & 5.9 \\
40 & 5.4 & 8.2 & 1.0 & 3.2 & + \\
60 & + & + & - & + & \\
Result & & & & & \\
\hline
\end{tabular}

Mutagenic index $=$ number of revertant colonies induced in the sample/number of spontaneous revertants in the negative control. $(+)=$ positive result; $(-)=$ negative result. $\mathrm{NT}=$ not tested. A drug was considered positive for mutagenicity only when the number of revertants was at least double the spontaneous yield: mutagenic index $\geq 2$. A significant dose-response was found by ANOVA $(P \leq 0.05)$ and a reproducible positive dose-response ( $\mathrm{P} \leq 0.01$ ) was present (ANOVA). Adapted from Ref. 25. 
morphine and 8-OASQ suggest that the two drugs might affect the formation of aversive memory through different molecular mechanisms. In fact, the finding that 8-OASQ fails to induce stereotyped behaviors (33) suggests that, whereas the neurobehavioral effects of apomorphine are probably mediated by activation of D1 and D2 dopamine receptors, the effects of 8-OASQ and possibly other oxidized metabolites of dopamine agonists are mediated by other mechanisms independent of dopamine receptor activation.

\section{Oxidative damage in the brain}

Given the possible involvement of apomorphine autoxidation products in neurotoxic activities, we evaluated the effects of the systemic administration of apomorphine and 8-OASQ on the oxidative parameters (thiobarbituric acid-reactive species, protein carbonyls and total radical-trapping antioxidants), and catalase and superoxide dismutase activities on the central nervous system (CNS) (34). Adult male CF-1 mice were treated with a systemic injection of apomorphine $(0.4,4.0$, or $40.0 \mathrm{mg} / \mathrm{kg})$ or 8 -OASQ $(0.4,4.0$, or $40.0 \mathrm{mg} / \mathrm{kg})$ and sacrificed by decapitation $24 \mathrm{~h}$ after treatment and the forebrains were collected to determine if apomorphine or 8-OASQ induced only transient alterations or could induce more sustained damage in the CNS. 8-OASQ appeared not to induce lipid peroxidation and only higher doses could induce protein carboxylation and a consumption of non-enzymatic antioxidant defenses, whereas apomorphine induced an increase in protein carboxylation and a consumption of non-enzymatic antioxidant defenses, as measured by the total radical-trapping antioxidant assay, at all doses tested (34). These results suggest that apomorphine biotransformation, and not necessarily 8-OASQ, may be related to some of the toxic effects of this drug. It is reasonable to suppose that, as is the case for dopamine oxidation, the oxidation of apomor- phine generates $\mathrm{H}_{2} \mathrm{O}_{2}$ or other free radicals that, in a favorable environment, could initiate oxidative stress.

Apomorphine and 8-OASQ induced an increase in catalase activity whereas superoxide dismutase activity was not affected at any dose tested. These data reinforce our suggestion that apomorphine biotransformation could produce $\mathrm{H}_{2} \mathrm{O}_{2}$, since it is well known that an increase in $\mathrm{H}_{2} \mathrm{O}_{2}$ content is a potent inductor of catalase activity (35). 8OASQ induces a dose-dependent catalase activation, and this could partially explain its less oxidant profile in our model. 8-OASQ probably either induces a more gradual increase in free radical production, which allows cell adaptation, or modulates intracellular signaling pathways that result in cell protection against oxidative stress. Supporting these hypotheses, an adaptive response has been observed in wild-type $S$. cerevisiae and in strains lacking antioxidant defenses following pretreatment of the cells with 8OASQ prior to exposure to a cytotoxic dose of $\mathrm{H}_{2} \mathrm{O}_{2}$ (25). None of the treatments modified superoxide dismutase activity in the CNS. The major inductor of superoxide activity is superoxide production. This supports the view that 8-OASQ does not generate superoxide, and that the principal ROS involved in apomorphine toxicity is $\mathrm{H}_{2} \mathrm{O}_{2}$.

Our results suggest that, in comparison to apomorphine, 8-OASQ seems to induce oxidative damage only at higher doses. In addition, 8-OASQ seems to up-regulate antioxidant systems.

\section{Brain DNA damage}

In order to evaluate a possible DNA damage induced by apomorphine and 8-OASQ in the brain we used the alkaline Comet assay in vivo (36) which detects DNA strand breaks, alkali-labile sites and incomplete excision repair events in individual cells $(37,38)$. There was a significant increase of DNA damage in mouse brain tissue 1 and 3 
h after treatment with 8-OASQ (Table 2). This result was expected since 8-OASQ displays a higher frameshift mutagenic activity, which stimulates DNA strand breaks, when compared to apomorphine (25). In addition, it is possible that 8-OASQ induces oxidative DNA damage mediated by its pro-oxidant effects, which were observed in cells of $S$. cerevisiae lacking antioxidant defenses. 8OASQ also produces oxidative mutagenesis in Escherichia coli WP2-derivative strains, in addition to the frameshift in S. typhimurium TA98 and TA97 (25). Probably OASQ displays these biological effects through usual redox reactions of quinones and semiquinones generating $\mathrm{H}_{2} \mathrm{O}_{2}$ or superoxide radicals, besides quinones and semiquinone radicals (29). These reactions could promote the increased formation of hydroxyl radicals that are able to induce mostly single-strand breaks and various species of oxidized purines and pyrimidines $(37,39)$.

In contrast to 8-OASQ, apomorphine did not induce significant damage to brain cells DNA at $1 \mathrm{~h}$ after treatment, although it induced a slight increase $3 \mathrm{~h}$ after treatment (36). It is possible that by $3 \mathrm{~h}$ after injection apomorphine had already been almost totally metabolized and these metabolites contributed to the increased DNA damage observed in brain cells. Indeed Battisti et al. (40) showed that brain apomorphine concentration significantly decreases between 1 and $2 \mathrm{~h}$ after injection. The 24-h sampling time showed a clear DNA repair response since no significant DNA damage could be found for either apomorphine or 8-OASQ (Table 2) (36).

The slight genotoxic effect of apomorphine in the brain is consistent with other studies suggesting that the drug has neurotoxic effects in vitro $(19,31)$. However, apomorphine may also play a role in preventing or slowing the rate of neurodegeneration associated with PD by scavenging iron and preventing dopamine-induced hydroxyl radical formation $(41,42)$. Accordingly, we have observed antimutagenic and antioxidant activities of apomorphine against t-BOOH and $\mathrm{H}_{2} \mathrm{O}_{2}$ on oxidative stress-sensitive strains such as the WP2-derivatives E. coli and TA102 S. typhimurium, and S. cerevisiae lacking antioxidant defenses (25).

These findings suggest that oxidation derivatives of apomorphine and possibly those of dopamine and L-DOPA clinically used for the treatment of PD might display genotoxic activities in brain when given in vivo. This finding should be taken into account when considering the possible neurotoxic effects of apomorphine and oxidized metabolites of dopamine agonists. Table 3 summarizes the results regarding the genotoxic and neurobiological activities of apomorphine and 8-OASQ.

\section{Neuroprotection}

Neuroprotection may be defined as preventing neuronal cell death and maintaining function without necessarily affecting the underlying biochemical mechanisms involved in pathogenesis. At the clinical level, this means stopping the progress of the disease. Neurorescue could be considered to be a mechanism reversing established metabolic

Table 2. Comet assay in brain tissue of mice treated with an intraperitoneal injection of vehicle, apomorphine, or 8-oxo-apomorphine-semiquinone (8-OASO), and sacrificed 1,3 or $24 \mathrm{~h}$ after injection.

\begin{tabular}{lccc}
\hline & Vehicle & Apomorphine $(40 \mathrm{mg} / \mathrm{kg})$ & 8-OASQ $(40 \mathrm{mg} / \mathrm{kg})$ \\
\hline $1 \mathrm{~h}$ & & & \\
$\mathrm{DI}$ & $16.0 \pm 2.2$ & $27.0 \pm 6.1$ & $146.5 \pm 14.4^{* *}$ \\
$\mathrm{DF}(\%)$ & $13.8 \pm 3.4$ & $20.5 \pm 4.1$ & $76.0 \pm 6.7^{* *}$ \\
$3 \mathrm{~h}$ & & & \\
$\mathrm{DI}$ & $19.0 \pm 2.5$ & $29.5 \pm 3.9$ & $80.3 \pm 20.6^{* *}$ \\
$\mathrm{DF}(\%)$ & $18.8 \pm 2.9$ & $29.5 \pm 3.9^{*}$ & $64.0 \pm 7.4^{* *}$ \\
$24 \mathrm{~h}$ & & & \\
$\mathrm{DI}$ & $7.3 \pm 3.9$ & $9.5 \pm 4.4$ & $7.5 \pm 2.4$ \\
$\mathrm{DF}(\%)$ & $7.3 \pm 3.9$ & $9.3 \pm 4.3$ & $7.5 \pm 2.4$ \\
\hline
\end{tabular}

$\mathrm{DI}=$ damage index - can range from 0 (completely undamaged, 100 cells $\times 0$ ) to 400 (maximum damaged, $100 \times 4)$. DF $(\%)=$ damage frequency - calculated based on number of cells with tail versus number of cells with no tails.

${ }^{*} \mathrm{P}<0.05,{ }^{*} \mathrm{P}<0.01$ compared to vehicle (Dunnett test). Adapted from Ref. 36. 
abnormalities and restoring normal neuronal function and survival. Clinically, this would result in an improvement of symptoms as well as a decrease in the progress of the disease $(43,44)$.

Antioxidants were the first drugs to be studied in an attempt to retard the progress of PD. However, the hypothesis that oxidative stress is associated with neuronal degeneration in PD has been tested in only a few clinical studies $(44,45)$. Selegiline and vitamin E (deprenyl and tocopherol antioxidative therapy of parkinsonism) were the first compounds to be evaluated as potential neuroprotective candidates for individuals with PD (46). Selegiline, but not vitamin E, delayed functional disability in untreated patients in the early phase of PD. However, it was not clear whether that was a symptomatic or an antioxidant effect (47). There are many alternative antioxidative approaches that may be considered in future clinical trials, including free-radical scavengers, glutathione-enhancing agents, ion chelators, and drugs that interfere with the oxidative metabolism of dopamine. Interestingly, the classic directly acting dopamine-receptor agonists may belong to the last group: by stimulating dopamine autoreceptors, these drugs reduce dopamine synthesis turnover and release, so that less L-DOPA is needed. In addition, some of these compounds have direct antioxidant effects (48).

Monoamine oxidase-B inhibitors were also tested in clinical trials based on their potential ability to slow neuronal degeneration by blocking the formation of free radicals derived from the oxidative metabolism of dopamine. Moreover, their neuroprotective effect goes beyond the ability to block monoamine oxidase- $\mathrm{B}$ and might involve up-regulation of anti-apoptotic molecules or binding to proapoptotic molecules (49).

Drugs that reduce glutamate release or

Table 3. Comparison of the cytotoxic, genotoxic and neurobiological effects of apomorphine (APO) and 8oxo-apomorphine-semiquinone (8-OASQ).

\begin{tabular}{|c|c|c|c|}
\hline \multirow[t]{2}{*}{ Activities } & \multicolumn{2}{|c|}{ Response } & \multirow[t]{2}{*}{ Reference } \\
\hline & APO & 8-OASO & \\
\hline Mutagenicity and cytotoxicity in vitro & & & 25 \\
\hline Frameshift mutagenesis (S. typhimurium TA98/TA97) & + & ++ & \\
\hline Oxidative mutagenesis (E. coli IC188/IC203) & - & + & \\
\hline Antimutagenesis (TA102/IC188/IC203) & + & NT & \\
\hline Cytotoxicity to S. cerevisiae (SOD, CAT, GSH, Yap1p-deficient strains) & - & + & \\
\hline Protection against oxidative damages in S. cerevisiae & + & - & \\
\hline Pro-oxidant effects in S. cerevisiae & - & + & \\
\hline Adaptive responses in S. cerevisiae & - & + & \\
\hline Neurobehavioral deficts & & & 32 \\
\hline Impairing effects on short-term retention of aversive training in rats & - & + & \\
\hline Impairing effects on long-term retention of aversive training in rats & + & + & \\
\hline Impaired habituation to a novel environment & + & - & \\
\hline Stereotypy induction & + & - & 33 \\
\hline DNA damage in brain cells of mice (Comet assay) & + & ++ & 36 \\
\hline Oxidative damage in brain of mice & & & 34 \\
\hline Catalase activity & + & + & \\
\hline Superoxide activity & - & - & \\
\hline Thiobarbituric acid reactive substances & - & - & \\
\hline Protein carbonyls & ++ & + & \\
\hline Total radical-trapping antioxidant parameter & ++ & + & \\
\hline
\end{tabular}


receptor interactions (glutamate toxicity blocking agents) also could be neuroprotective compounds. Glutamate has been implicated as one of the most important neurotransmitters involved in the functional organization of the cortical-basal ganglion-thalamic and subthalamic connections. It probably plays a major role in the development of dyskinesias and response fluctuations. It is believed that the beneficial effect of pallidotomy and deep brain stimulation arises from the interference with glutamatergic circuits, and that early surgical procedures may even have neuroprotective effects (50). Extensive studies are in progress in order to determine whether riluzole (a drug that blocks glutamate release) or similar agents have potential neuroprotective effects or symptomatic effects on disabling motor complications (reviewed in Ref. 44).

Neuroprotection of the dopaminergic system was observed in animal experiments using growth factors. Among the most promising factors is glial-derived neurotrophic factor that has been shown to restore dopaminergic function in N-methyl-4-phenyl1,2,3,6-tetrahydropyridine (MPTP)-treated monkeys (51). The discovery of mutations in several genes in familial cases with PD has contributed to the understanding of the mechanisms involved in the pathogenesis of PD. Two proteins with mutations have been extensively studied: $\alpha$-synuclein and parkin. Both proteins probably aggregate and accumulate within Lewy bodies (52). Therefore, a promising neuroprotective strategy will use agents that prevent abnormal protein accumulation and aggregation.

Probably, the most effective mode of a protective therapy consists of a combination of agents with distinct pharmacological effects as opposed to monotherapy. An important point is also that this cocktail of drugs should be advocated in the early stages of the disease to exert maximum clinical efficacy, and for this reason determination of early markers of PD is a prerequisite. PET and
SPECT studies might be of help in the evaluation of disease progression as well as of the rate of progression following different modes of treatment $(45,53)$.

The main challenge facing those involved in the management of patients with PD is the development of a neuroprotective therapy that can be administrated early in the course of the disease and that can slow, stop, or reverse (neurorescue) disease progression (44).

The protective effect of a drug can be expressed in terms of decreasing damage to cellular structures, such as lipids, proteins or nucleic acid (RNA and DNA). Results obtained in vitro and in vivo using models of oxidative brain damage support the hypothesis that dopaminergic agonists can be neuroprotective $(54,55)$. Knowledge about the degeneration of dopamine-containing neurons and the effects of neuroprotective drugs have come from animal and cell culture studies that employ the neurotoxins MPTP, 6hydroxydopamine, methamphetamine, rotenone, and N-methyl-R-salsolinol, which replicate many of the neurochemical and anatomical characteristics of the parkinsonian syndrome in rodents, primates and other species (56). These neurotoxins are thought to produce their dopaminergic neurotoxicity via generation of ROS, liberation of ferritin iron in the substantia nigra pars compacta, depletion of reduced glutathione, and inhibition of mitochondrial complex I.

Experimental studies have demonstrated that treatment with a dopaminergic agonist prevented nigrostriatal cell loss in those animal models of PD $(57,58)$. It is suggested that the neuroprotective effect of these drugs is based on their action as antioxidants. The defense strategies required of any potential antioxidant therapy include the ability of the drug to cross the blood-brain barrier, to scavenge ROS or their precursors, to inhibit ROS formation, and to up-regulate endogenous antioxidant defenses. Other explanations may include a levodopa-sparing effect, stimula- 
tion of dopaminergic autoreceptors leading to decreased dopamine release, a receptormediated anti-apoptotic effect, and inhibition of excitotoxicity mediated by overactivity of the subthalamic nucleus.

In vitro studies have shown that apomorphine is an iron chelator (4), reduces the oxidation of polyunsaturated fatty acids (23) and is a potent free radical scavenger that protects pheochromocytoma $\mathrm{PC} 12$ cells from oxidative stress induced by $\mathrm{H}_{2} \mathrm{O}_{2}$ and 6hydroxydopamine (54). Furthermore, apomorphine up-regulates nerve growth factor and glial cell line-derived neurotrophic factor synthesis in cultured mouse astrocytes (59).

In addition, many animal models have demonstrated a neuroprotective effect of apomorphine. Pretreatment of mice with apomorphine followed by MPTP results in protection of nigrostriatal neurodegeneration (4). This protection is expressed by preventing the MPTP-induced depletion of striatal dopamine and tyrosine hydroxylase content. Apomorphine dose-dependently protects against methamphetamine-induced striatal dopamine loss and reduction of TH activity in the rat striatum (60). Apomorphine was found to be a potent hydroxyl radical scavenger in an experimental model involving iron and dopamine perfusion in the rat striatum (42). Continuous subcutaneous infusion of apomorphine rescues nigrostriatal dopaminergic neurons from toxicity induced by MPTP in mice (41). The neuroprotective effect of apomorphine appears to be based on its antioxidant and free radical scavenging properties and does not seem to be related to its dopamine agonist activity.
Recently, we have observed a significant decrease of DNA damage induced by the carcinogenic agent methyl methanesulfonate in mouse brain tissue pretreated with 8OASQ, suggesting a neuroprotective effect of the agent (Flores DG, Picada JN, Roesler $\mathrm{R}$ and Henriques JAP, unpublished results). In that model, apomorphine was unable to protect brain cells against methyl methanesulfonate damage, maybe due to the fact that methyl methanesulfonate is not an oxidant agent. Furthermore, the results indicated that 8-OASQ could induce other adaptive responses besides those related to oxidative damages.

\section{Final remarks}

The genotoxic activity of apomorphine in bacteria might be related to its ability to intercalate into DNA and/or to its pro-oxidant effects or generation of superoxide radicals during autoxidation, hence promoting frameshift mutations without inducing oxidative mutagenesis. This idea is supported by the higher mutagenic frameshift induced by 8-OASQ, a more aromatic structural compound which favors intercalation into DNA. The neurotoxic effects of apomorphine have been correlated with its autoxidation products such as superoxide radical, $\mathrm{H}_{2} \mathrm{O}_{2}$ and quinone and semiquinone compounds. It is possible that 8-OASQ is one of these cytotoxic products, since 8-OASQ induced oxidative mutagenesis in bacteria, adaptive responses in S. cerevisiae, as well as DNA damage and alterations of oxidative parameters in the mouse brain.

\section{References}

1. Gotz ME, Freyberger A \& Riederer P (1990). Oxidative stress: a role in the pathogenesis of Parkinson's disease. Journal of Neural Transmission, 29 (Suppl): 241-249.

2. Ben-Shachar DP, Riederer P \& Youdim MBH (1991). Iron-melanin interaction and lipid peroxidation: implications for Parkinson's disease. Journal of Neurochemistry, 57: 1609-1614.
3. Fahn S \& Cohen G (1992). The oxidant stress hypothesis in Parkinson's disease: Evidence supporting it. Annals of Neurology, 32: 804-812.

4. Youdim MBH, Grünblatt E \& Mandel S (1999). The pivotal role of iron in NF-KB activation and nigrostriatal dopaminergic neurodegeneration. Prospects for neuroprotection in Parkinson's disease with 
iron chelators. Annals of the New York Academy of Sciences, 890: 7-25.

5. Ebadi M, Srinivasan SK \& Baxi MD (1996). Oxidative stress and antioxidant therapy in Parkinson's disease. Progress in Neurobiology, 48: 1-19.

6. Jenner $P$ (1998). Oxidative mechanisms in nigral cell death in Parkinson's disease. Movement Disorders, 13: 24-34.

7. Sian J, Dexter DT, Lees AJ, Daniel S, Agid Y, Javoy-Agid F, Jenner P \& Marsden CD (1994). Alterations in glutathione levels in Parkinson's disease and other neurodegenerative disorders affecting basal ganglia. Annals of Neurology, 36: 348-355.

8. Dexter DT, Holley AE, Lees AJ, Agid F, Agid Y, Jenner P \& Marsden CD (1994). Increased levels of hydroperoxides in the parkinsonian substantia nigra: An HPLC and ESR study. Movement Disorders, 9: 92-97.

9. Spencer JP, Jenner A, Aruoma OI, Evans PJ, Kaur H, Dexter DT, Jenner P, Lees AJ, Marsden DC \& Halliwell B (1994). Intense oxidative DNA damage promoted by L-dopa and its metabolites. Implications for neurodegenerative disease. FEBS Letters, 353: 246-250

10. Dragunow M, MacGibbon GA, Lawlor P, Butterworth N, Connor B, Henderson C, Walton M, Woodgate A, Hughes P \& Faull RL (1997). Apoptosis, neurotrophic factors and neurodegeneration. Reviews in the Neurosciences, 8: 223-265.

11. Bianchi G \& Landi M (1985). Determination of apomorphine in rat plasma and brain by high-performance liquid chromatography with electrochemical detection. Journal of Chromatography, 338: 230235.

12. Di Chiara G \& Gessa GL (1978). Pharmacology and neurochemistry of apomorphine. Advances in Pharmacology and Chemotherapy, 15: 87-160.

13. Corboy DL, Wagner ML \& Sage JL (1995). Apomorphine for motor fluctuations and freezing in Parkinson's disease. Annals of Pharmacotherapy, 29: 282-288.

14. Pzedborski S, Levivier M, Raftopoulos C, Naini AB \& Hildebrand J (1995). Peripheral and central pharmacokinetics of apomorphine and its effect on dopamine metabolism in humans. Movement Disorders, 10: 28-36.

15. Graham DG (1978). Oxidative pathways for catecholamines in the genesis of neuromelanin and cytotoxic quinones. Molecular Pharmacology, 14: 633-643.

16. Bindolli A, Rigobello MP \& Deeble DJ (1992). Biochemical and toxicological properties of the oxidation products of catecholamines. Free Radical Biology and Medicine, 13: 391-405.

17. Segura-Aguillar J, Metodiewa D \& Welch CJ (1998). Metabolic activation of dopamine o-quinones to o-semiquinones by NADPH cytochrome $\mathrm{P} 450$ reductase may play an important role in oxidative stress and apoptotic effects. Biochimica et Biophysica Acta, 1381: $1-6$.

18. Smythies JR (1997). Oxidative reactions and schizophrenia: A review-discussion. Schizophrenia Research, 24: 357-364.

19. El-Bachá RS, Daval J, Koziel V, Netter P \& Minn A (2001). Toxic effects of apomorphine on rat cultured neurons and glial C6 cells, and protection with antioxidants. Biochemical Pharmacology, 66: 73-85.

20. Costa C, Bertazzo A, Allegri G, Toffano G, Curcuruto O \& Traldi P (1992). Melanin biosynthesis from dopamine. II. A mass spectrometric and collisional spectroscopic investigation. Pigment Cell Research, 5: 122-131.

21. Sit SY (2000). Dopamine agonists in the treatment of Parkinson's disease - past, present and future. Current Pharmaceutical Design,
6: 1211-1248.

22. Ubeda A, Montesinos C, Payá M \& Alcaraz MJ (1993). Iron reducing and free radical scavenging properties of apomorphine and some related benzylisoquinolines. Free Radical Biology and Medicine, 15: 159-167.

23. Gassen M, Glinka Y, Pinchasi B \& Youdim MBH (1996). Apomorphine is a highly potent free radical scavenger in rat brain mitochondrial fraction. European Journal of Pharmacology, 308: 219-225.

24. Khromov-Borisov NN, Picada JN \& Henriques JAP (2000). Dose finding in the Ames Salmonella assay. Mutation Research, 453: 3544.

25. Picada JN, Maris AF, Ckless K, Salvador M, Khromov-Borisov NN \& Henriques JAP (2003a). Differential mutagenic, antimutagenic and cytotoxic responses induced by apomorphine and its oxidation product, 8-oxo-apomorphine-semiquinone, in bacterial and yeast. Mutation Research, 539: 29-41.

26. Suter W \& Matter-Jaeger I (1984). Genotoxicity of apomorphine and various catecholamines in the Salmonella mutagenicity (Ames test) and in tests for primary DNA damage using repair-deficient $B$. subtilis strains (Rec assay). Mutation Research, 137: 17-28.

27. Tadaki S, Nozaka T, Yamada S, Ishino M, Morimoto I, Tanaka A \& Kunitomo J (1992). Clastogenicity of apomorphine alkaloids in vitro. Journal of Pharmacobio-Dynamics, 15: 501-512.

28. Cheng H-Y, Strope E \& Adams RN (1979). Electrochemical studies of the oxidation pathways of apomorphine. Analytical Chemistry, 51: 2243-2246.

29. Kalyanaraman B (2000). Characterization of o-quinone radicals in biological systems. Methods in Enzymology, 186: 333-343.

30. Liu J \& Mori A (1993). Monoamine metabolism provides an antioxidant defense in the brain against oxidant- and free radical-induced damage. Archives of Biochemistry and Biophysics, 302: 118-127.

31. El-Bacha RS, Netter P \& Minn A (1999). Mechanisms of apomorphine cytotoxicity towards rat glioma C6 cells: protection by bovine serum albumin and formation of apomorphine-protein conjugates. Neuroscience Letters, 263: 25-28.

32. Picada JN, Schroder N, Izquierdo I, Henriques JAP \& Roesler R (2002). Differential neurobehavioral deficits induced by apomorphine and its oxidation product, 8-oxo-apomorphine-semiquinone, in rats. European Journal of Pharmacology, 443: 105-111.

33. Picada JN, Henriques JAP \& Roesler R (2003). An oxidized form of apomorphine fails to induce stereotypy. Schizophrenia Research, 63: 199-200.

34. Moreira JC, Dal-Pizzol F, Bonatto F, da Silva EG, Flores DG, Picada JN, Roesler R \& Henriques JAP (2003). Oxidative damage in brains of mice treated with apomorphine and its oxidized derivative. Brain Research, 992: 246-251.

35. Halliwell B \& Gutteridge JMC (1999). Free Radicals in Biology and Medicine. 3rd edn. Oxford University Press, New York.

36. Picada JN, Flores DG, Zettler CG, Marroni NP, Roesler R \& Henriques JAP (2003). DNA damage in brain cells of mice treated with an oxidized form of apomorphine. Molecular Brain Research, 114: 80-85.

37. Collins A, Ai-Guo M \& Duthie SJ (1995). The kinetics of repair of oxidative DNA damage (strand breaks and oxidized pyrimidines) in human cells. Mutation Research, 336: 69-77.

38. Tice RR, Agurell E, Anderson D, Burlinson B, Hartmann A, Kobayashi H, Miyamae Y, Rojas E, Ryu J-C \& Sasaki YF (2000). Single cell gel/ comet assay: guidelines for in vitro and in vivo genetic toxicology testing. Environmental and Molecular Mutagenesis, 35: 206-221.

39. Brozmanová J, Dudás A \& Henriques JAP (2001). Repair of oxidative DNA damage - an important factor reducing cancer risk. Neo- 
plasma, 48: 85-93.

40. Battisti JJ, Uretsky NJ \& Wallace LJ (2000). Importance of environmental context in the development of amphetamine- or apomorphine-induced stereotyped behavior after single and multiple doses. Pharmacology, Biochemistry, and Behavior, 66: 671-677.

41. Battaglia G, Busceti CL, Cuomo L, Giorgi FS, Orzi F, Blasi A De, Nicoletti F, Ruggieri S \& Fornai F (2002). Continuous subcutaneous infusion of apomorphine rescues nigrostriatal dopaminergic terminals following MPTP injection in mice. Neuropharmacology, 42: 367-373.

42. Chen Y-K, Lin H-C, Liu J-C \& Wan F-J (2001). Potent, hydroxyl radical-scavenging effect of apomorphine with iron and dopamine perfusion in rat striatum. Brain Research, 896: 165-168.

43. Schapira AHV (2002). Neuroprotection and dopamine agonists. Neurology, 58 (Suppl 1): S9-S18.

44. Schapira AHV \& Olanow CW (2004). Neuroprotection in Parkinson disease. Journal of the American Medical Association, 291: 358364.

45. Brooks DJ (2000). Monitoring neuroprotection and restorative therapies in Parkinson's disease. Journal of Neural Transmission, 60 (Suppl): 125-137.

46. Hunter C \& Jankovic J (1999). Double-blind, placebo-controlled study to assess safety and efficacy of riluzole as a neuroprotective drug in patients with early untreated Parkinson's disease. Neurology, 52 (Suppl 2): 214-215.

47. Olanow WC (2002). The role of dopamine agonists in the treatment of early Parkinson's disease. Neurology, 58 (Suppl 1): S33-S41.

48. Dunnett SB \& Björklund A (1999). Prospects for new restorative and neuroprotective treatments in Parkinson's disease. Nature, 399: A32-A39.

49. Maruyama W, Akao Y, Youdim MB \& Naoi M (2000). Neurotoxins induce apoptosis in dopamine neurons: protection by $\mathrm{N}$-propargylamine- $1^{\circledR}$ and (S)-aminoindan, rasagiline and TV1022. Journal of Neural Transmission, 60 (Suppl): 171-186.

50. Conley SC \& Kirchner JT (1999). Medical and surgical treatment of Parkinson's disease. Strategies to slow symptom progression and improve quality of life. Postgraduate Medicine, 106: 41-44.
51. Rioux L, Frohna PA, Joyce JN \& Schneider JS (1997). The effects of chronic levodopa treatment on pre and post-synaptic markers of dopaminergic function in striatum of parkinsonian monkeys. Movement Disorders, 12: 148-158.

52. Shimura H, Schlossmacher MG, Hattori N et al. (2001). Ubiquitination of a new form of alpha-synuclein by parkin from brain: implication for Parkinson's disease. Science, 293: 263-269.

53. Whone AL, Watts RL, Stoessl AJ et al. (2003). Slower progression of Parkinson's disease with ropinirole versus levodopa: the REALPET study Annals of Neurology, 54: 93-101.

54. Gassen M, Gross A \& Youdim MB (1998). Apomorphine enantiomers protect cultured pheochromocytoma (PC12) cells from oxidative stress induced by $\mathrm{H}_{2} \mathrm{O}_{2}$ and 6-hydroxydopamine. Movement Disorders, 13: 661-667.

55. O'Neill M, Hicks C, Ward M, Cardwell G, Reymann J, Allain H \& Bentue-Ferrer D (1998). Dopamine D2 receptor agonists protect against ischemia-induced hippocampal neurodegeneration in global cerebral ischemia. European Journal of Pharmacology, 352: 37-46.

56. Mandel S, Grunblatt E, Levites Y, Maor G \& Youdim MBH (2003). Genes and oxidative stress in parkinsonism: cDNA microarray studies. Advances in Neurology, 91: 123-132.

57. Grünblatt E, Mandel S, Maor G \& Youdim MB (2001). Effects of Rand S-apomorphine on MPTP-induced nigrostriatal dopamine neuronal loss. Journal of Neurochemistry, 77: 146-156.

58. Grünblatt E, Mandel S, Maor G \& Youdim MB (2001). Gene expression analysis in N-methyl-4-phenyl-1,2,3,6-tetrahydropyridine mice model of Parkinson's disease using cDNA microarray: Effect of Rapomorphine. Journal of Neurochemistry, 78: 1-12.

59. Ohta M, Mizuta I, Ohta K, Nishimura M, Minuta E, Hayashi K \& Kuno S (2000). Apomorphine up-regulates NGF and GDNF synthesis in cultural mouse astrocytes. Biochemical and Biophysical Research Communications, 272: 18-22.

60. Fornai F, Battaglia G, Gesi M, Orzi F, Nicoletti F \& Ruggieri S (2001). Dose-dependent protective effects of apomorphine against methamphetamine-induced nigrostriatal damage. Brain Research, 898: 27-35 